\title{
Two New Bromotyrosine-Derived Metabolites from the Sponge Psammaplysilla purpurea
}

\author{
Thirumani Venkateshwar Goud, ${ }^{a}$ Masuna Srinivasulu, ${ }^{a}$ Vanimireddy Lakshmi Niranjan Reddy, ${ }^{a}$ \\ Adelli Vijender Reddy, ${ }^{a}$ Tadikamalla Prabhakar Rao, ${ }^{a}$ Dondapati Srujan Kumar, ${ }^{b}$ \\ Upadhyayula SurYanaramyana Murty, ${ }^{b}$ and Yenamandra VenKateSWARLu ${ }^{*, a}$ \\ ${ }^{a}$ Natural Products Laboratory, Organic Division-I, Indian Institute of Chemical Technology; and ${ }^{b}$ Biology Division, \\ Indian Institute of Chemical Technology; Hyderabad-500 007, India. Received February 24, 2003; accepted May 8, 2003
}

\begin{abstract}
Two new bromotyrosine-derived metabolites $(1,2)$ have been isolated along with the known compounds $3,5-$ dibromo-4-methoxyphenylacetonitrile, 3-bromo-4-methoxyphenylacetonitrile, 3-bromo-4-hydroxyphenylacetonitrile, 1-hydroxyuracil, 1-methoxyhemibastadin 2, purpuramine $H$ and a steroid $5 \alpha, 8 \alpha$-epidioxycholest-6-en-3 $\beta$-ol from the sponge Psammaplysilla purpurea. Compounds 1 and 2 were characterized by interpretation of their spectral data. The antibacterial activity of these compounds is summarized.
\end{abstract}

Key words sponge; Psammaplysilla purpurea; purpuramine; antibacterial activity

Sponges of the family Verongiidea have produced a series of antibiotics, which may be considered as metabolites of halogenated tyrosine metabolites. ${ }^{1,2)}$ During the course of our search for biologically active compounds from the marine organisms, we have investigated the sponge Psammaplysilla purpurea CARTER (Aplysinellidae), collected from the Mandapam coast in Southern India during February 2002. A literature survey revealed that the genus Psammaplysilla has yielded several bromotyrosine-derived metabolites, namely psammaplysins, ${ }^{3)}$ purpuramines, ${ }^{4)}$ macrocyclic bastadins and hemibastadins. ${ }^{5,6)}$

The $\mathrm{CH}_{2} \mathrm{Cl}_{2}-\mathrm{MeOH}(1: 1)$ extract of the sponge Psammaplysilla purpurea was partitioned between water and EtOAc. The EtOAc soluble portion was concentrated under reduced pressure and subjected to gel filtration (Sephadex LH-20) followed by silica gel column chromatography eluting with a step gradient of hexane-EtOAc mixtures, to $\mathrm{MeOH}$, to afford known compounds 3,5-dibromo-4methoxyphenyl acetonitrile, 3-bromo-4-methoxyphenylacetonitrile, ${ }^{7)}$ 3-bromo-4-hydroxyphenyl acetonitrile, ${ }^{8)}$ 1-hydroxyuracil, ${ }^{9)}$ 1-methoxyhemibastadin $2,{ }^{10)}$ purpuramine $\mathrm{H}^{4)}$ and a steroid $5 \alpha, 8 \alpha$-epidioxycholest-6-en- $3 \beta$-ol, ${ }^{11)}$ and two new compounds, $\mathbf{1}$ and 2. Compounds $\mathbf{1}$ and $\mathbf{2}$ were obtained as a mixture that resisted separation by Sephadex LH-20 partition chromatography or normal silica gel chromatography using a variety of solvent systems. The mixture was then rechromatographed on a reversed-phase $\left(\mathrm{C}_{18}\right)$ HPLC column using $20 \%$ aqueous $\mathrm{MeOH}$ as eluent, where upon two new compounds purpuramine K (1) and L (2) were isolated, and the isolated compounds were tested for antibacterial activity against Staphylococcus, Bacillus (gram positive), Chromobaterium, Klebsiella and Pseudomonas (gram negative).

\section{Results and Discussion}

Purpuramine K (1) was obtained as an optically inactive white solid (yield $0.058 \%$ on dry wt basis), mp $190-195^{\circ} \mathrm{C}$. Its molecular formula was established as $\mathrm{C}_{24} \mathrm{H}_{28} \mathrm{~N}_{3} \mathrm{O}_{6} \mathrm{Br}_{3}$ by high resolution (HR)-FAB-MS, which showed molecular cluster ion $(\mathrm{M}+\mathrm{H})^{+}$peaks at $m / z$ 692, 694, 696 and 698 in $1: 2: 2: 1$ ratio suggesting the presence of 3 bromine atoms. The IR spectrum showed bands at 3404, 2926, 1675 and $1494 \mathrm{~cm}^{-1}$ which indicated the presence of hydroxyl and

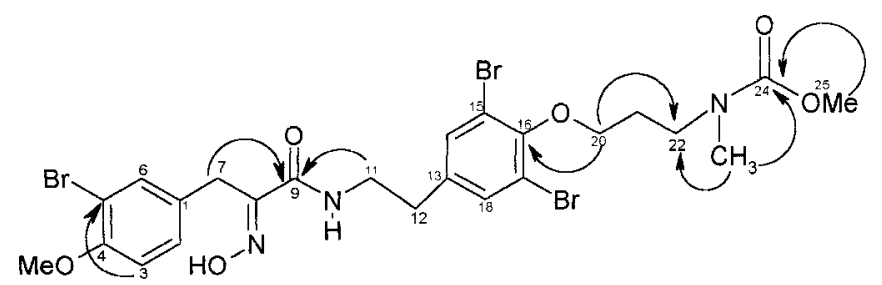

1

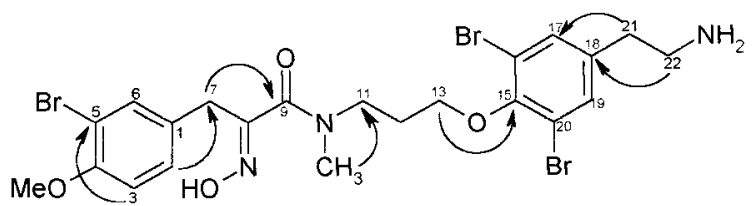

2

Selected HMBC correlations

amide groups. Its ${ }^{1} \mathrm{H}-\mathrm{NMR}$ spectrum (Table 1) displayed four aromatic signals ascribable to the presence of two benzene rings viz. a 1,3,4-trisubstituted benzene $\delta 7.12(1 \mathrm{H}, \mathrm{dd}, 2-\mathrm{H})$, $\delta 6.97(1 \mathrm{H}, \mathrm{d}, 3-\mathrm{H})$ and $\delta 7.38(1 \mathrm{H}, \mathrm{d}, 6-\mathrm{H})$ and a $1,3,4,5$ tetra substituted benzene with a peak at $\delta 7.46(2 \mathrm{H}, \mathrm{s}, 14,18-$ $\mathrm{H})$. Further its ${ }^{1} \mathrm{H}-\mathrm{NMR}$ spectrum displayed signals for six methylene groups at $\delta 3.91(2 \mathrm{H}, \mathrm{t}, 20-\mathrm{H}), \delta 3.72(2 \mathrm{H}, \mathrm{s}, 7-$ $\mathrm{H}), \delta 3.38(2 \mathrm{H}, \mathrm{t}, 22-\mathrm{H}), \delta 3.35(2 \mathrm{H}, \mathrm{t}, 11-\mathrm{H}), \delta 2.73(2 \mathrm{H}, \mathrm{t}$, $12-\mathrm{H})$ and $\delta 2.03(2 \mathrm{H}, \mathrm{m}, 21-\mathrm{H})$; an aromatic methoxyl group at $\delta 3.79(3 \mathrm{H}, \mathrm{s})$, an ester methyl at $\delta 3.57(3 \mathrm{H}, \mathrm{s})$, a $\mathrm{N}-\mathrm{Me}$ at $\delta 2.83(3 \mathrm{H}, \mathrm{s})$ and $\mathrm{D}_{2} \mathrm{O}$ exchangeable protons at $\delta$ $7.99(1 \mathrm{H}, \mathrm{t}, \mathrm{NH})$ and at $\delta 11.82(1 \mathrm{H}$, br s, N-OH). The foregoing spectral data revealed that compound $\mathbf{1}$ belongs to the purpuramine $\mathrm{A}-\mathrm{I}^{4}$ ) class. The linear connectivity of the methylene groups was established by the ${ }^{1} \mathrm{H}-{ }^{1} \mathrm{H}$ correlation spectroscopy (COSY) spectrum. In the ${ }^{1} \mathrm{H}-{ }^{1} \mathrm{H}$ COSY spectrum the signal at $\delta 2.03$ showed correlations with an oxygen bearing methylene at $\delta 3.91$ and with a methylene at $\delta 3.38$, and two mutually coupled methylene signals at $\delta 2.73$ and $\delta$ 3.35. From the study of its ${ }^{1} \mathrm{H}-$ and ${ }^{13} \mathrm{C}-\mathrm{NMR}$ spectra, the three methyl signals at $\delta 3.79, \delta 3.57$ and $\delta 2.83$ were assigned to an aromatic methoxyl $\left[\delta_{\mathrm{C}} 56.2\right.$ (q)], to an ester 
Table $1 .{ }^{13} \mathrm{C}-(125 \mathrm{MHz}),{ }^{1} \mathrm{H}-\mathrm{NMR}(500 \mathrm{MHz})$ and HMBC (500 MHz) Data for Compounds $\mathbf{1}^{a)}$ and $\mathbf{2}^{b)}$

\begin{tabular}{|c|c|c|c|c|c|c|}
\hline \multirow{2}{*}{ Position } & \multicolumn{3}{|c|}{ Compound 1} & \multicolumn{3}{|c|}{ Compound 2} \\
\hline & $\delta_{\mathrm{C}}$ (mult.) & $\delta_{\mathrm{H}}$ & HMBC & $\delta_{\mathrm{C}}$ (mult.) & $\delta_{\mathrm{H}}$ & HMBC \\
\hline 1 & $130.4(\mathrm{~s})$ & & & $131.7(\mathrm{~s})$ & & \\
\hline 2 & $129.1(\mathrm{~d})$ & $7.12(1 \mathrm{H}, \mathrm{dd}, J=2.0,8.0)$ & $\mathrm{C} 4, \mathrm{C} 6, \mathrm{C} 7$ & $130.4(\mathrm{~d})$ & $7.02(1 \mathrm{H}, \mathrm{dd}, J=2.0,8.0)$ & $\mathrm{C} 6, \mathrm{C} 7$ \\
\hline 3 & $112.6(\mathrm{~d})$ & $6.97(1 \mathrm{H}, \mathrm{d}, J=8.0)$ & $\mathrm{C} 1, \mathrm{C} 5$ & $113.2(\mathrm{~d})$ & $6.78(1 \mathrm{H}, \mathrm{d}, J=8.0)$ & $\mathrm{C} 1, \mathrm{C} 5$ \\
\hline 4 & $153.8(\mathrm{~s})$ & & & $155.7(\mathrm{~s})$ & & \\
\hline 5 & $110.2(\mathrm{~s})$ & & & $112.5(\mathrm{~s})$ & & \\
\hline 6 & 133.0 (d) & $7.38(1 \mathrm{H}, \mathrm{d}, J=2.0)$ & $\mathrm{C} 2, \mathrm{C} 4, \mathrm{C} 5$ & 135.0 (d) & $7.44(1 \mathrm{H}, \mathrm{d}, J=2.0)$ & $\mathrm{C} 4, \mathrm{C} 7$ \\
\hline 7 & $27.8(\mathrm{t})$ & $3.72(2 \mathrm{H}, \mathrm{s})$ & $\mathrm{C} 2, \mathrm{C} 6, \mathrm{C} 8, \mathrm{C} 9$ & $28.1(\mathrm{t})$ & $3.76(2 \mathrm{H}, \mathrm{s})$ & $\mathrm{C} 1, \mathrm{C} 2, \mathrm{C} 6, \mathrm{C} 8, \mathrm{C} 9$ \\
\hline 8 & $151.8(\mathrm{~s})$ & & & $152.7(\mathrm{~s})$ & & \\
\hline 9 & $163.2(\mathrm{~s})$ & & & $165.5(\mathrm{~s})$ & & \\
\hline 11 & $41.1(\mathrm{t})$ & $3.35(2 \mathrm{H}, \mathrm{t}, J=7.0)$ & $\mathrm{C} 9, \mathrm{C} 12, \mathrm{C} 13$ & $48.7(\mathrm{t})$ & $3.15(2 \mathrm{H}, \mathrm{t}, J=7.2)$ & $\mathrm{C} 9, \mathrm{C} 12, \mathrm{C} 13, \mathrm{~N}-\mathrm{CH}_{3}$ \\
\hline 12 & $34.0(\mathrm{t})$ & $2.73(2 \mathrm{H}, \mathrm{t}, J=7.0)$ & $\mathrm{C} 11, \mathrm{C} 13, \mathrm{C} 14, \mathrm{C} 18$ & $29.0(\mathrm{t})$ & $2.15(2 \mathrm{H}, \mathrm{tt}, J=7.2,6.0)$ & $\mathrm{C} 11, \mathrm{C} 13$ \\
\hline 13 & $139.0(\mathrm{~s})$ & & & $71.5(\mathrm{t})$ & $4.08(2 \mathrm{H}, \mathrm{t}, J=6.0)$ & $\mathrm{C} 11, \mathrm{C} 12$ \\
\hline 14 & $132.9(\mathrm{~d})$ & $7.46(2 \mathrm{H}, \mathrm{s})$ & $\mathrm{C} 12, \mathrm{C} 15, \mathrm{C} 16$ & - & & \\
\hline 15 & $117.2(\mathrm{~s})$ & & & $152.2(\mathrm{~s})$ & & \\
\hline 16 & $150.7(\mathrm{~s})$ & & & $118.9(\mathrm{~s})$ & & \\
\hline 17 & $117.2(\mathrm{~s})$ & & & $134.3(\mathrm{~d})$ & $7.36(2 \mathrm{H}, \mathrm{s})$ & $\mathrm{C} 15, \mathrm{C} 16, \mathrm{C} 19, \mathrm{C} 21$ \\
\hline 18 & 132.9 (d) & $7.46(2 \mathrm{H}, \mathrm{s})$ & $\mathrm{C} 12, \mathrm{C} 16, \mathrm{C} 17$ & $139.9(\mathrm{~s})$ & & \\
\hline 19 & - & & & $134.3(\mathrm{~d})$ & $7.36(2 \mathrm{H}, \mathrm{s})$ & $\mathrm{C} 15, \mathrm{C} 17, \mathrm{C} 20, \mathrm{C} 21$ \\
\hline 20 & $71.0(\mathrm{t})$ & $3.91(2 \mathrm{H}, \mathrm{t}, J=6.0)$ & $\mathrm{C} 16, \mathrm{C} 21, \mathrm{C} 22$ & $118.9(\mathrm{~s})$ & & \\
\hline 21 & $28.0(\mathrm{t})$ & $2.03(2 \mathrm{H}, \mathrm{m})$ & $\mathrm{C} 20, \mathrm{C} 22$ & $35.5(\mathrm{t})$ & $2.72(2 \mathrm{H}, \mathrm{t}, J=7.8)$ & $\mathrm{C} 17, \mathrm{C} 18, \mathrm{C} 19, \mathrm{C} 22$ \\
\hline 22 & $40.6(\mathrm{t})$ & $3.38(2 \mathrm{H}, \mathrm{t}, J=6.8)$ & $\mathrm{C} 20, \mathrm{C} 21, \mathrm{C} 24, \mathrm{~N}-\mathrm{Me}$ & $41.5(\mathrm{t})$ & $3.45(2 \mathrm{H}, \mathrm{t}, J=7.8)$ & $\mathrm{C} 18, \mathrm{C} 21$ \\
\hline 24 & $156.5(\mathrm{~s})$ & & & - & & \\
\hline 25 & $52.3(q)$ & $3.57(3 \mathrm{H}, \mathrm{s})$ & $\mathrm{C} 24$ & - & & \\
\hline $\mathrm{NH}$ & & $7.99(1 \mathrm{H}, \mathrm{t}, J=6.0)$ & & - & & \\
\hline $\mathrm{N}-\mathrm{Me}$ & $41.4(q)$ & $2.83(3 \mathrm{H}, \mathrm{s})$ & $\mathrm{C} 22, \mathrm{C} 24$ & $34.4(\mathrm{q})$ & $2.60(3 \mathrm{H}, \mathrm{s})$ & $\mathrm{C} 11$ \\
\hline $\mathrm{OMe}$ & $56.2(\mathrm{q})$ & $3.79(3 \mathrm{H}, \mathrm{s})$ & $\mathrm{C} 4$ & $57.0(\mathrm{q})$ & $3.78(3 \mathrm{H}, \mathrm{s})$ & $\mathrm{C} 4$ \\
\hline
\end{tabular}

a) DMSO- $d_{6}$. b) $\mathrm{CD}_{3} \mathrm{OD}$.

methyl of a carbamate ${ }^{12)}\left[\delta_{\mathrm{C}} 156.5(\mathrm{~s}), \delta 52.3(\mathrm{q})\right]$ and to a methyl on nitrogen bearing carbamate ester [ $\delta_{\mathrm{C}} 41.4$ (q)] respectively. The structure of compound $\mathbf{1}$ was established by the study of heteronuclear multiple bond connectivity (HMBC) and nuclear Overhauser effect spectroscopy (NOESY) correlations. In its HMBC spectrum the C-22 methylene protons at $\delta 3.38(2 \mathrm{H}, \mathrm{t}, J=6.8 \mathrm{~Hz})$ showed correlations with C-20, C-24 and to a $N$-methyl at $\delta_{\mathrm{C}} 41.4$ (q) respectively. Further, the $N$-methyl at $\delta_{\mathrm{H}} 2.83\left(\delta_{\mathrm{C}} 41.4\right)$ showed correlation with carbamate carbonyl at $\delta_{\mathrm{C}} 156.5$ (s). The C11 methylene protons at $\delta 3.35(2 \mathrm{H}, \mathrm{t}, J=7 \mathrm{~Hz})$ showed correlation with $\mathrm{C}-9$ and $\mathrm{C}-13$ carbons. In the NOESY spectrum, the benzylic methylene signal at $\delta 2.73(2 \mathrm{H}, \mathrm{t}, J=7 \mathrm{~Hz}$, $12-\mathrm{H})$ showed correlation with two aromatic protons at $\delta$ $7.46(2 \mathrm{H}, \mathrm{s}, 14,18-\mathrm{H})$, the methoxyl signal at $\delta 3.79(3 \mathrm{H}, \mathrm{s})$ showed correlation with an aromatic proton at $\delta 6.97(1 \mathrm{H}, \mathrm{d}$, $J=8 \mathrm{~Hz}, 3-\mathrm{H})$, the methyl signal at $\delta 2.83(3 \mathrm{H}, \mathrm{s})$ showed correlation with a methylene group at $\delta 3.38(2 \mathrm{H}, \mathrm{t}$, $J=6.8 \mathrm{~Hz}, 22-\mathrm{H})$ connected to methyl carbamate. The foregoing spectral data suggested that compound $\mathbf{1}$ is methyl carbamate of purpuramine I. Thus the structure of purpuramine $\mathrm{K}$ was established as $\mathbf{1}$.

Purpuramine L (2) was obtained as an optically inactive white solid (yield $0.08 \%$ on dry wt basis), $\mathrm{mp} 175-178^{\circ} \mathrm{C}$. Its molecular formula was established as $\mathrm{C}_{22} \mathrm{H}_{26} \mathrm{~N}_{3} \mathrm{O}_{4} \mathrm{Br}_{3}$ by HR-FAB-MS, which showed molecular cluster ion $(\mathrm{M}+\mathrm{H})^{+}$ peaks at $\mathrm{m} / \mathrm{z} 633,635,637$ and 639 in $1: 2: 2: 1$ ratio suggesting the presence of 3 bromine atoms. The IR spectrum showed bands at 3350,1670,1200, 1137 and $720 \mathrm{~cm}^{-1}$, which indicated the presence of hydroxyl and amide groups. The ${ }^{1} \mathrm{H}-\mathrm{NMR}$ spectrum of compound $\mathbf{2}$ displayed 11 signals for 26 protons and its ${ }^{13} \mathrm{C}$-NMR spectrum showed the pres- ence of N-Me and $\mathrm{O}-\mathrm{Me}\left[\delta_{\mathrm{H}} 2.60\left(\delta_{\mathrm{C}} 34.4\right) ; \delta_{\mathrm{H}} 3.78\left(\delta_{\mathrm{C}}\right.\right.$ 57.0)]. The ${ }^{1} \mathrm{H}$ - and ${ }^{13} \mathrm{C}-\mathrm{NMR}$ spectra of compound 2 closely resemble those of purpuramines A-I. ${ }^{4)}$ Its ${ }^{1} \mathrm{H}-\mathrm{NMR}$ spectrum displayed four aromatic signals due to two benzene rings viz., a 1, 3, 4 tri substituted benzene $[\delta 7.02(1 \mathrm{H}, \mathrm{dd}, 2-$ $\mathrm{H}), 6.78(1 \mathrm{H}, \mathrm{d}, 3-\mathrm{H}), 7.44(1 \mathrm{H}, \mathrm{d}, 6-\mathrm{H})]$ and a $1,3,4,5$ tetra substituted benzene $[\delta 7.36(2 \mathrm{H}, \mathrm{s}, 17,19-\mathrm{H})]$. Further, its ${ }^{1}$ H-NMR displayed signals for six methylenes (Table 1). The linear connectivity of these methylene groups was established by following ${ }^{1} \mathrm{H}-{ }^{1} \mathrm{H}$ COSY spectral data. A methylene group proton signal at $\delta 2.15(2 \mathrm{H}, \mathrm{tt}, 12-\mathrm{H})$ showed correlation with a methylene bearing an oxygen atom at $\delta 4.08(2 \mathrm{H}$, $\mathrm{t}, 13-\mathrm{H})$ and also with a methylene bearing amide nitrogen at $\delta 3.15(2 \mathrm{H}, \mathrm{t}, 11-\mathrm{H})$. There were also, two mutually coupled methylene group signals at $\delta 3.45(2 \mathrm{H}, \mathrm{t}, 22-\mathrm{H})$ and $\delta 2.72$ $(2 \mathrm{H}, \mathrm{t}, 21-\mathrm{H})$. Of the two methyl groups present in the molecule, one was assigned to an aromatic methoxyl group at C-4 $[\delta 3.78(3 \mathrm{H}, \mathrm{s})]$. The other methyl group which resonated at $\delta 2.60(3 \mathrm{H}, \mathrm{s})$ was placed on the amide nitrogen of the molecule due to its NOESY correlations with the methylene protons at $\delta 3.15(2 \mathrm{H}, \mathrm{t}, \mathrm{H}-11)$ and by consideration of its ${ }^{13} \mathrm{C}$ NMR chemical shift. ${ }^{4)}$ The ${ }^{1} \mathrm{H}$ - and ${ }^{13} \mathrm{C}-\mathrm{NMR}$ values of the $\mathrm{N}-\mathrm{Me}$ group in the propyl ether side chain of purpuramine $\mathrm{G}$ and I were reported at $\delta_{\mathrm{H}} 2.76(3 \mathrm{H}, \mathrm{s}), \delta_{\mathrm{C}} 27.7(\mathrm{q})$ and $\delta_{\mathrm{H}}$ $2.76(3 \mathrm{H}, \mathrm{s}), \delta_{\mathrm{C}} 27.7(\mathrm{q})$ respectively. ${ }^{4)}$ However in the case of compound 2 its ${ }^{13} \mathrm{C}$ signal appeared $\left[\delta_{\mathrm{C}} 34.4(\mathrm{q})\right]$ substantially further downfield due to its presence on amide nitrogen. ${ }^{16)}$ The foregoing spectral data was further corroborated by its HMBC correlations. In the HMBC spectrum of $\mathbf{2}$, the $\mathrm{C}-11$ methylene protons at $\delta 3.15(2 \mathrm{H}, \mathrm{t}, J=7.2 \mathrm{~Hz})$ showed correlations with C-9, C-12, C-13 and N-Me. The C-21 methylene protons at $\delta 2.72(2 \mathrm{H}, \mathrm{t}, J=7.8 \mathrm{~Hz})$ showed corre- 
Table 2. Antibacterial Activity of Compounds $\mathbf{1}$ and $\mathbf{2}$

\begin{tabular}{|c|c|c|c|c|c|c|c|c|}
\hline & \multirow{3}{*}{$\begin{array}{c}\text { Name of } \\
\text { the organism }\end{array}$} & \multicolumn{6}{|c|}{ Concentration $\left.(\mu \mathrm{g} / \text { disk })^{a}\right)$} & \multirow{3}{*}{$\begin{array}{l}\text { Kanamycin }^{b)} \\
(30 \mu \mathrm{g} / \text { disk })\end{array}$} \\
\hline & & \multicolumn{3}{|c|}{1} & \multicolumn{3}{|c|}{2} & \\
\hline & & 25 & 50 & 100 & 25 & 50 & 100 & \\
\hline \multirow[t]{3}{*}{ Gram positive } & S. aureus & 10 & 10 & 12 & 10 & 14 & 16 & 10 \\
\hline & B. subtilis & 8 & 10 & 12 & 10 & 14 & 16 & 18 \\
\hline & B. sphaericus & - & 9 & 11 & 7 & 12 & 14 & 20 \\
\hline \multirow[t]{3}{*}{ Gram negative } & C. violaceum & 7 & 8 & 11 & 10 & 13 & 15 & 17 \\
\hline & K. aerogenes & 10 & 11 & 13 & 7 & 10 & 12 & 15 \\
\hline & P. aeruginosa & 10 & 12 & 14 & 12 & 16 & 18 & 27 \\
\hline
\end{tabular}

a) Values represent zones inhibition in $\mathrm{mm} / \mathrm{dia}$. b) Positive control.

lation with $\mathrm{C}-17, \mathrm{C}-18, \mathrm{C}-19$ and $\mathrm{C}-22$ respectively. From the foregoing spectral data the structure of purpuramine $\mathrm{L}$ was established as $\mathbf{2}$.

The upfield ${ }^{13} \mathrm{C}-\mathrm{NMR}$ chemical shifts of C-7 in compounds 1 and $2\left[\delta_{\mathrm{C}} 27.8(\mathrm{t})\right.$ and $\left.\delta_{\mathrm{C}} 28.1(\mathrm{t})\right]$ suggests an $E$ configuration for the oxime ${ }^{13)}$ (the corresponding in the case of a $(Z)$ oxime is $\left.>35 \mathrm{ppm}^{14)}\right)$.

Antibacterial Activity The antibacterial activity was assayed by disk susceptibility tests according to the NCCLS (Wayne, 1997), ${ }^{15)}$ Inocula were adjusted to 0.5 McFarland turbidity. Excess moisture was allowed to absorb for $10 \mathrm{~min}$. before applying dried disks containing the compound. Compounds were dissolved in sterile dimethyl sulfoxide (DMSO), which did not influence the growth of bacteria. Kanamycin was used as a positive control according to the standard method. The test plates were incubated at $37^{\circ} \mathrm{C}$ and zones of inhibition were recorded after $24 \mathrm{~h}$. Purpuramines K (1) and L (2) were tested (Table 2) against gram positive bacteria Staphylococcus aureus (ATCC \#9144), Bacillus subtilis (ATCC \#6051), Bacillus sphaericus (ATCC \#14577) and gram negative bacteria Chromobaterium violaceum (ATCC \#12472), Klebsiella aerogenes (ATCC \#15380), Pseudomonas aeruginosa (ATCC \#25619).

Compound $\mathbf{2}$ is highly active against $S$. aureus, B. subtilis and $C$. violaceum, moderately active against $B$. sphaericus, $K$. aerogenes, and $P$. aeruginosa. Compound $\mathbf{1}$ is moderately active against all the organisms. Interestingly, gram positive bacteria are highly susceptible to compound $\mathbf{2}$ when compared to the positive control kanamycin.

\section{Experimental}

General Optical rotations were measured on a JASCO DIP-370 polarimeter. UV and IR spectra were recorded on Shimadzu-240 and PerkinElmer $240-\mathrm{C}$ instruments, respectively. ${ }^{1} \mathrm{H}-(500 \mathrm{MHz})$ and ${ }^{13} \mathrm{C}-\mathrm{NMR}$ $(125 \mathrm{MHz})$ spectra were recorded on a Varian Gemini $500 \mathrm{MHz}$ spectrometer using tetramethyl silane (TMS) as internal standard. Chemical shifts are reported in parts per million and coupling constants $(J)$ are expressed in Hertz. Mass spectra were recorded on a VG Auto Spec-M instrument. Preparative scale HPLC was performed using a Supelcosil $\mathrm{C}_{18}$ column ( $60 \AA, 12 \mu \mathrm{m}, 25 \mathrm{~cm} \times 21.2 \mathrm{~mm})$.

Animal Material The sponge Psammaplysilla purpurea was collected from the Mandapam coast in the Gulf of Mannar, Tamilnadu, India, during February 2002. A voucher specimen (IIC-441) is on deposit at the National Institute of Oceanography, Goa, India.

Extraction and Isolation The freshly collected sponge specimens were soaked in $\mathrm{MeOH}$ at the site of collection and kept in $\mathrm{MeOH}$ until workup. The sponge Psammaplysilla purpurea $(1.5 \mathrm{~kg})$ was extracted with $1: 1$ $\mathrm{CH}_{2} \mathrm{Cl}_{2}-\mathrm{MeOH}(3 \times 31)$ at room temperature. The combined extracts including the initial methanol extract were filtered, and the solvent was removed under reduced pressure to give a predominantly aqueous suspension, which was partitioned between water and EtOAc. The EtOAc soluble portion was again concentrated under reduced pressure to give a dark brown gummy mass $(10 \mathrm{~g})$. This crude extract $(10 \mathrm{~g})$ was subjected to gel filtration chromatography (Sephadex LH-20, 1:1 $\mathrm{CH}_{2} \mathrm{Cl}_{2}-\mathrm{MeOH}, 47 \mathrm{~mm} \times 820 \mathrm{~mm}$ ) collecting 30 fractions $(25 \mathrm{ml}$ each) followed by silica gel chromatography of selected fractions using a step gradient of hexane to hexane-ethyl acetate mixtures to $\mathrm{MeOH}$, to yield 3,5-dibromo-4-methoxyphenylacetonitrile ( $40 \mathrm{mg}$ ), 3-bromo-4-methoxyphenylacetonitrile $(60 \mathrm{mg})$, 3-bromo-4-hydroxyphenylacetonitrile $(100 \mathrm{mg}), 1$-hydroxyuracil $(30 \mathrm{mg}), 5 \alpha, 8 \alpha$-epidioxycholest-6-ene-3 $\beta$-ol $(150 \mathrm{mg})$, compound $\mathbf{1}(7 \mathrm{mg})$ and compound $\mathbf{2}(10 \mathrm{mg})$. The fractions eluted with hexane-EtOAc $(40: 60)$ which could not be purified by silica gel chromatography are combined and purified on a preparative reversed-phase $\left(\mathrm{C}_{18}\right)$ HPLC column [MeOH- $\left.\mathrm{H}_{2} \mathrm{O}(80: 20)\right]$, (flow rate of $5 \mathrm{ml} / \mathrm{min}$ ) to give compounds $\mathbf{1}$ and $\mathbf{2}$.

Purpuramine K (1): Optically inactive white solid (7 mg), mp 190$195^{\circ} \mathrm{C}$; IR (KBr) $v_{\max } 3404,2926,1675$ and $1494 \mathrm{~cm}^{-1}$; UV (MeOH) $\lambda_{\max }$ ( $\log \varepsilon$ ) 217.8 (3.65), 280.2 (4.78); for ${ }^{1} \mathrm{H}-\mathrm{NMR}\left(500 \mathrm{MHz}\right.$, DMSO- $d_{6}$ ) and ${ }^{13} \mathrm{C}$-NMR $\left(125 \mathrm{MHz}\right.$, DMSO- $d_{6}$ ) see Table 1; positive FAB-MS $\mathrm{m} / \mathrm{z} 692$, 694,696 and $698(\mathrm{M}+\mathrm{H})^{+}$in a $1: 2: 2: 1$ ratio; positive HR-FAB-MS $m / z$ observed $691.9726[\mathrm{M}+\mathrm{H}]^{+}$(Calcd for $\mathrm{C}_{24} \mathrm{H}_{28} \mathrm{~N}_{3} \mathrm{O}_{6}{ }^{79} \mathrm{Br}_{3} \mathrm{~m} / \mathrm{z}$ 694.2150, $\Delta-2.2 \mathrm{mmu}$ ).

Purpuramine L (2): Optically inactive white solid $(10 \mathrm{mg}), \mathrm{mp} 175-$ $178^{\circ} \mathrm{C}$; IR (KBr) $v_{\max } 3350,1670,1200,1137$ and $720 \mathrm{~cm}^{-1}$; UV (MeOH) $\lambda_{\max }(\log \varepsilon) 214.5$ (5.12), 280.0 (4.02), for ${ }^{1} \mathrm{H}-\mathrm{NMR}$ (500 MHz, $\mathrm{CD}_{3} \mathrm{OD}$ ), ${ }^{13} \mathrm{C}-\mathrm{NMR}\left(125 \mathrm{MHz}, \mathrm{CD}_{3} \mathrm{OD}\right)$ see Table 1; positive FAB-MS $\mathrm{m} / \mathrm{z} 633,635$, 637 and $639(\mathrm{M}+\mathrm{H})^{+}$in a $1: 2: 2: 1$ ratio; positive HR-FAB-MS $\mathrm{m} / \mathrm{z}$ observed $632.9650[\mathrm{M}+\mathrm{H}]^{+}$(Calcd for $\mathrm{C}_{22} \mathrm{H}_{26} \mathrm{~N}_{3} \mathrm{O}_{4}{ }^{79} \mathrm{Br}_{3} \mathrm{~m} / z$ 635.7964, $\Delta-2.8 \mathrm{mmu}$ ).

Acknowledgments We are thankful to Dr. P. A. Thomas, Central Marine Fisheries Research Institute, Vizhinjam, Thiruvananthapuram, India, for identifying the sponge, the Department of Ocean Development, New Delhi, India, for financial assistance, the Director IICT, and Dr. J. S. Yadav for their encouragement, and CSIR, New Delhi for providing fellowships to T .V. G., V. L. N. R. and A. V. R.

\section{References and Notes}

1) Bergquist P. R., Wells R. J., "Marine Natural Products: Chemical and Biological Perspectives," Vol. 5, ed. by Scheuer P. J., Academic Press, New York, 1985, pp. 1-50.

2) Faulkner D. J., Nat. Prod. Rep., 18, 1-49 (2001), and literature cited in previous reviews.

3) Rotem M., Carmely S., Kashman Y., Loya Y., Tetrahedron, 39, 667676 (1983).

4) Yagi H., Matsunaga S., Fusetani N., Tetrahedron, 49, 3749-3754 (1993).

5) Carney J. R., Scheuer P. J., Kelly-Borges M., J. Nat. Prod., 56, 153157 (1993).

6) Pettit G. R., Butler M. S., Bass C. G., Doubek D. L., Williams M. D., Schmidt J. M., Pettit R. K., Hooper J. N. A., Tackett L. P., Filiatrault M. J., J. Nat. Prod., 58, 680 - 688 (1995).

7) Venkateswarlu Y., Ramdas C., J. Nat. Prod., 58, 1087-1088 (1995).

8) Quinoa E., Crews P., Tetrahedron Lett., 28, 3229-3232 (1987).

9) Ohigashi H., Kaji M., Sakaki M., Koshimizu K., Phytochemistry, 28, 1365-1368 (1989). 
10) Pettit G. R., Butler M. S., Williams M. D., Filiatrault M. J., Pettit K. R., J. Nat. Prod., 59, 927-934 (1996).

11) Gunatilaka A. A. L., Gopichand Y., Schmitz F. J., Djerassi C., J. Org. Chem., 46, 3860-3866 (1981).

12) Venkateswarlu Y., Venkatesham U., Rama Rao M., J. Nat. Prod., 62, 893-894 (1999).

13) Arabshahi L., Schmitz F. J., J. Org. Chem., 52, 3584-3586 (1987).
14) Jurek J., Yoshida W., Scheuer P., J. Nat. Prod., 56, 1609-1612 (1993).

15) Wayne P. A., "National Committee for Clinical Laboratory Standards Performance Standards for Antimicrobial Disk Susceptibility Tests," 6th ed., Approved standards M2-A6. NCCLS, 1997.

16) Biemann K., "Tables of Spectral Data for Structure Determination of Organic Compounds," 2nd ed., Springer-Verlag, Berlin, 1989. 